\title{
Augmented Reality for Human Needs: An Ontology
}

\author{
Manal A. YAHYA ${ }^{\mathrm{a}, 1}$ and Ajantha DAHANAYAKE ${ }^{\mathrm{a}}$ \\ ${ }^{a}$ Lappeenranta University of Technology, Lappeenranta, Finland
}

\begin{abstract}
Augmented Reality is a display and interaction method of future computing. It augments digital information in real environments in text, audio, image, or video formats. Augmented reality can be more effective if supported by knowledge about human needs. Basic human needs are finite in number, and with the right methods, they are detectable or predictable. This research develops an ontology that describes the structure and relations between the elements of augmented reality, context information, and human needs, with the ultimate goal of developing a robust conceptual model. Ontology development is a knowledgedriven approach used to represent data and reasoning. This paper focuses on linking the aforementioned concepts to enable correct data representation and reasoning. The research approach, process used, and the evaluation of the ontology is presented as well.
\end{abstract}

Keywords. Human needs, augmented reality, context-awareness, ontology, satisfiers, conceptual model

\section{Introduction}

An Ontology is a notion that has origins in Latin (onto + logy), which means the science of being or existence. The term is borrowed from philosophy and used in the field of computer science. Thomas Gruber set its definition to be "a specification of a representational vocabulary for a shared domain of discourse- definitions of classes, relations, functions, and other objects- is called an ontology" [1]. Ontologies are commonly used in semantic web technologies to ease computers' understanding and manipulation of internet data. The semantic web is built on a set of languages and technologies: The Extensible Markup Language (XML) provides the syntax. The Resource Description Framework (RDF) represents information about resources. The RDF schema enables a taxonomical organization, and the Web Ontology Language (OWL) provides expressive constructs to extend the RDF schema [2].

This paper presents an ontology that describes the use of Augmented Reality in the satisfaction of human needs.

\footnotetext{
${ }^{1}$ Corresponding Author, Manal Yahya; E-mail: manal.ahmad@gmail.com.
} 


\subsection{Augmented Reality}

An Augmented Reality (AR) system embodies the following properties [3]:

- It enhances real environments by adding virtual objects.

- It works in real-time and provides interactivity.

- It provides the correct placement of virtual objects within the environment.

AR has many application areas such as education and learning [4], entertainment and gaming [5], food and beverage industry [6], health care [7], manufacturing [8], museums [9], space exploration [10], and tourism [11].

\subsection{Human Needs}

There is a difference between needs, desires, and satisfiers [12]:

- Need: the most fundamental requirement and the base for desires and satisfiers.

- Desire: personal and intentional, two possible desires may fulfill a specific need; however, it is a personal preference to choose one over the other.

- Satisfier: objects or states that fulfill needs or desires.

This research focuses on needs and satisfiers rather than desires to keep the case general to all users. There are many theories of human needs. The goal of using MaxNeef's model [13] in this research is to provide a base for recognizing human needs rather than claiming that it is the only valid model.

\subsection{Problem Definition and Motivation}

The study of human needs has roots in psychology, economics, philosophy, sociology, anthropology, and social sciences, which indicates the importance of needs, and their satisfaction in human life. Despite that fact, there is still a shortage of incorporating human needs in information systems and tools [14]. Augmented reality is a growing field that may benefit from the utilization of human needs. A.K. Dey [15] defines context awareness as "A system that uses context to provide relevant information and services to the user, where relevancy depends on the user's task." This concept allows augmented reality applications to be adaptive [16]; nevertheless, we argue that incorporating the concept of human needs leverages the pervasiveness of context-aware systems. Hence the motive to study the development of augmented reality applications with a concentration on human needs detection and satisfaction. Previous studies focused on the development of a conceptual model to address this motive [17] [18]. The characteristics of conceptual models, according to [19] are:

- Conceptual models have conceptual semantics

- They are ontologically grounded

- They make an ontological commitment

These characteristics reflect the base in which ontologies provide support for the development of conceptual models and the use of a foundational ontology to advance conceptual modeling [20].

From another perspective, some of the reasons that drive the effort of ontology development are [21]:

- It provides a shared understanding of the structure of information between people or software agents.

- It analyzes domain knowledge and separating it from operational knowledge. 
- It allows domain knowledge reuse.

- It explicitly defines domain assumptions.

Based on the above reasoning, this paper looks into the development of an ontology that combines the concepts of augmented reality, human needs' satisfaction, and the detection of those needs from context and user information to support conceptual modeling. Previous studies on ontologies included a representation of needs as they are found in various theories of human needs [14], an ontology focusing only on Max-Neef's model of human needs [22], context modeling [23], and developing augmented reality based on ontological context awareness [16].

The contribution and novelty of this research involve developing a vocabulary that conceptualizes the relationship between humans, their needs, existing satisfiers, and their possible representation in augmented reality, with the ultimate goal of providing augmented reality experiences to users according to their detected needs. This ontology should enhance and enrich our conceptual model, and enable the development of models and systems based on it.

In addition to the representation of needs, this research requires building relations with other elements affecting them, such as the trigger of a need, the context in which it occurs, and the possible services that satisfy those needs.

This research follows a bottom-up approach to defining the cycle of needs. The cycle starts with a balanced state, a trigger elicits a need which is then detected, a satisfier is matched with the need detected, which is then accepted or rejected, and the user provides feedback at the end of the cycle, and a state of balance is retained. All the elements in this cycle must be represented clearly in the ontology under development.

This paper is divided as follows: Section 2 explains the research approach used in the development of the ontology. Section 3 discusses related work. Section 4 details the steps of ontology development and the outcomes. Then, section 5 presents the evaluation of the ontology. Finally, the discussion and the conclusion are in sections 6 and 7.

\section{Research Approach}

The methodologies described in [24] and [21] are chosen and combined to develop the Augmented Reality for human needs ontology. Those research papers describe detailed steps in ontology development.

In [24] Methontology (a term coined by the researchers), the ontology lifecycle is defined by some states and activities (Fig. 1), these activities represent the method to develop the ontology. Below is a list of activities and important notes for each activity.

\section{Methontology Phases [24]}

1. Specification: In this phase, the developers produce an ontology specification document that includes the purpose, level of formality, and the scope of the ontology under development.

2. Knowledge Acquisition: This step may be performed parallel to the specification phase and continues until not needed; knowledge can be collected from many sources, including books, experts, and research papers.

3. Conceptualization: this phase requires the development of a conceptual model based on the information from the specification document. The model should 
demonstrate the problem and its solution. To aid the development of the model, the authors suggest creating a Glossary of Terms (GT), including concepts, properties, instances, and verbs.

4. Integration: the authors suggest using previously built ontologies to derive terms and their definitions and descriptions. They also recommend building an integration document that describes the meaning of each term, the ontology it was derived from, and its name in the conceptual model.

5. Implementation: this activity requires the utilization of an ontology development environment or editor to build the complete ontology.

6. Evaluation: the ontology must be evaluated through verification and validation following predefined techniques based on the specification document.

7. Documentation: in addition to the publication resulting from building the ontologies, thorough documentation aids in keeping the ontology, maintaining, and reusing it.

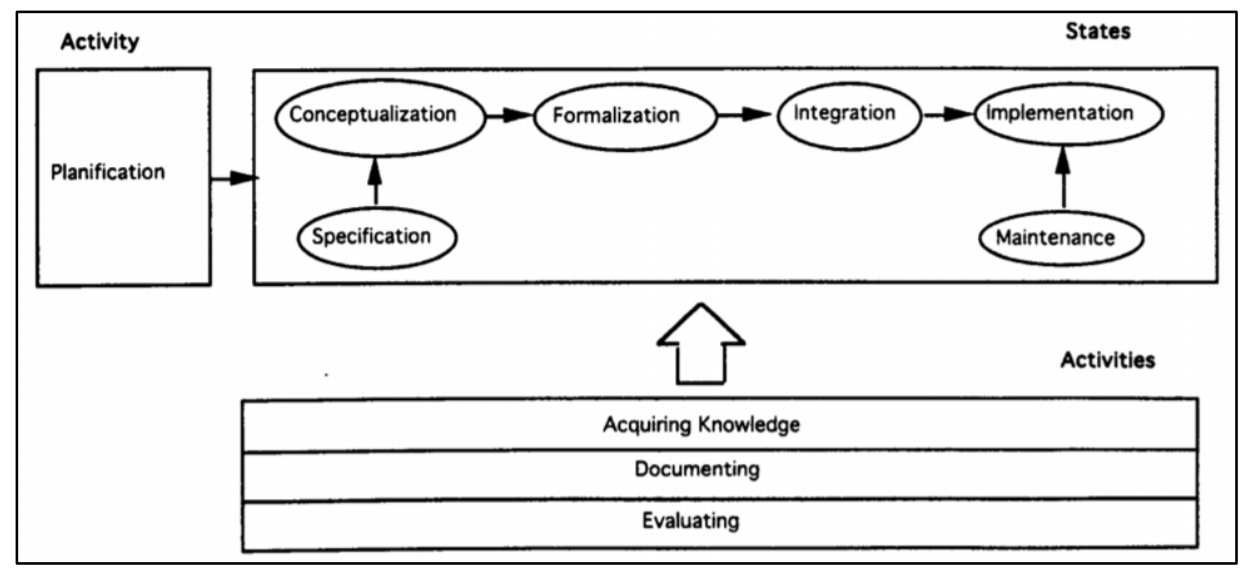

Figure 1. States and Activities of Ontology development [24]

The research on ontology development in [21] defines the following steps for the ontology development process:

1. Determine the domain and scope of ontology.

2. Consider reusing existing ontologies.

3. Enumerate important terms in the ontology.

4. Define the classes and class hierarchy.

5. Define the properties of classes-slots.

6. Define the facets of the slots.

7. Create instances.

\section{Related Works}

This section reviews previous research on the topics at the intersection of augmented reality, human needs, context awareness, and ontology development. 
The research presented in [16] studies adding adaptiveness to augmented reality by using ontologies, and aims to represent and analyze context. The defining factor in creating adaptivity is user activity. Four main elements compose the structure of the context model ontology: user ontology, device ontology, physical environment ontology, and service ontology. The developed AR application provides the required information in a three steps process:

1. The mobile device reads the URI object reference.

2. The device requests the relevant information from the server that returns an RDF describing the location of all relevant elements.

3. The user views a relevant augmented reality scene based on the situation.

The researchers developed a prototype that enables reading of Quick Response (QR) codes and the use of Near-Field Communication (NFC) technology. They provide an Ambient Assisted Living (AAL) scenario to evaluate the proposed system.

In [22], Dsouza presents the Fundamental Human Needs (FHN) ontology, based on Max-Neef's Fundamental Human Needs theory [13]. The research defines the main entities in the conceptual model as Agent, Role, Need, Satisfier, and Existential Category. As a method to further describe the satisfier, he portrayed the existential category to be a categorization of the satisfier rather than the need. The author used the Methontology [24] method to develop the ontology and provided a verification and validation approach to evaluate it.

An ontology-based model for human activity representation in smart homes [25], proposes a model developed using the $\mathrm{NeON}$ methodology [26], and it consists of connected ontologies that fall in one of three categories: user ontologies, smart home context ontologies, and Activity of Daily Life (ADL) ontologies. The authors provided a case describing a scenario of an elder person living in a smart home environment; they applied the developed ontology on the case scenario for evaluation. The researchers identified two main approaches to model activity in the smart home environment:

- Data-Driven approach:

Learns activity models using data mining and machine learning methods.

- Advantages: good at processing uncertain knowledge, and can handle long-term temporal data.

- Disadvantages: suffers from the cold start problem, and lacks the reusability in mixed systems.

- Knowledge-Driven approach (including Ontology development):

It uses knowledge engineering methods and representation formalisms.

- Advantage: effective representation abilities and reasoning methods for heterogeneous data.

A study in [27] describes the use of cultural heritage ontology to provide location information in specific cultural sites. The researchers developed an application that provides contextual information in mobile augmented reality. They used the Korea Cultural Heritage Data Model (KCHDM) to collect and display contextual information which has five super-classes: actor, event, thing, time-span, and place. It also has eightyfour properties that connect the classes.

The researchers in [14] pinpointed the limited use of human needs concepts in information systems and the lack of needs assessment tools in computing. They developed an ontology called OPENEED and incorporated need theories and need assessment methods. OPENEED is composed of a core ontology (Fig. 2) that describes fundamental human needs theories, and extensions that describe adjectives to describe needs, and assessment methods. The researches performed a need assessment study in a 
Viennese district local community to validate the developed ontology. The collection of ontologies in OPENEED has a set of rules in the Semantic Web Rule Language (SWRL)[28]. The authors identified five main applications of the ontology:

1. Enables publishing of related studies as "self-contained datasets."

2. Allows querying the results from single or multiple studies that make comparative analysis possible.

3. Enriches the data collected in studies by using SWRL rules.

4. Enables tracking the origin of assessments and evaluations, whether by individuals, experts, or communities.

5. Allows representation of different sizes of data due to the modular structure of the ontology.

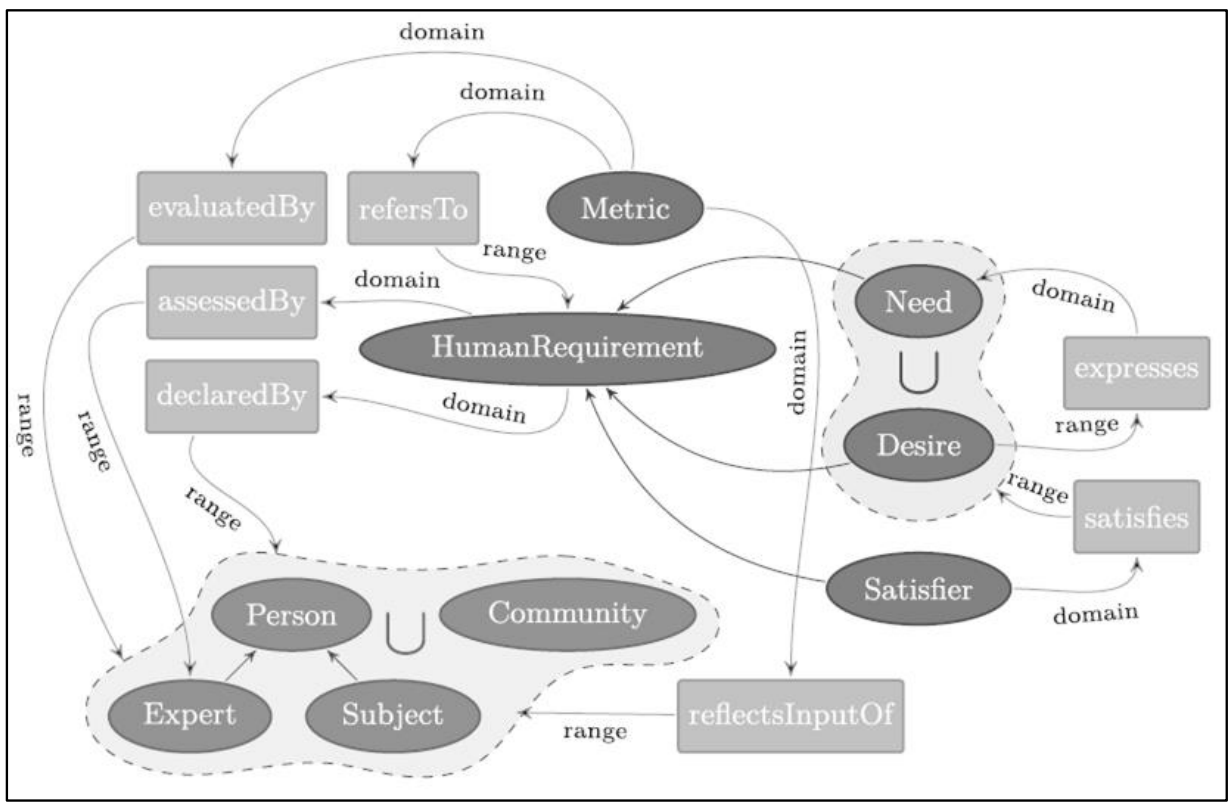

Figure 2 Outline of Core-OPEENEED Ontology [14]

\section{Ontology Development}

This section provides details on creating an ontology that is capable of representing the information of a user, his/her needs, context, AR experiences as services, and the relationship between these concepts.

\subsection{Specification: Determine the Domain and Scope of the Ontology}

Several questions must be answered to determine the domain and scope [21]:

- "What is the domain that the ontology will cover? 
- For what we are going to use the ontology?

- For what types of questions, the information in the ontology should provide answers?

- Who will use and maintain the ontology?"

The domain defined for the "Augmented Reality for Human Needs" ontology includes the information needed to detect human needs, the representation of those needs, and elements of augmented reality experiences that represent the satisfiers or aids in the satisfaction of those needs. This ontology may be used for applications that match AR satisfiers with human needs when they arise. The ontology is used and maintained initially by the authors of this research.

As an additional help to answer the third question, the following competency questions are proposed so that a system using this ontology should be able to answer:

- Which human need is currently active for a user?

- What augmented reality experience can satisfy a specific need?

- What is the current internal state of the user?

A significant task in the ontology development process is to create an Ontology Requirement Specification Document (ORSD), which describes the purpose of the ontology, its uses, and users, and the requirements it should realize [29].

Table 1 Ontology Requirement Specification Document

\section{AR for Human Need Ontology Requirements Specification Document}

\section{Purpose \\ The purpose of creating the ontology is to provide a knowledge model for the augmented reality domain that responds to various human needs and fulfills them. \\ 2 Scope \\ The ontology should focus on the application development and representation part of the described system.}

\section{Implementation Language}

\section{$\mathrm{RDF} / \mathrm{OWL}$}

\section{$4 \quad$ Intended End-Users}

User 1. Application developer aiming to develop a system that detects human needs and matches it with AR satisfiers.

User 2. The Person who uses the application to find needs satisfiers.

User 3. The Company providing services as satisfiers.

User 4. Health care provider monitoring a user's change in health

\section{Intended Uses}

Use 1. Develop an application interface and background that reflect the ontology and match needs and satisfiers.

Use 2. Create a profile, monitor needs' triggers and view possible satisfiers

Use 3. Create AR satisfiers for various human needs 
Use 4. Provide monitored health information

\section{Ontology Functional Requirements}

FR 1. The Ontology proposed must support user modeling. Static information, as well as dynamic context information about the user, must be supported.

FR 2. The ontology must support a human needs theory model, in this case, Max-Neef's model of human needs.

7 Pre-Glossary Terms

Augmented Reality, Human needs, satisfaction, balance, triggers, feedback

\subsection{Consider Reusing existing Ontologies}

After a deliberate search for ontologies that encompass the concepts of augmented reality and human needs, below are research papers that provide similar ideas and inspire the development of our proposed ontology:

1. FHN- Cloud-based Ontology solution for conceptualizing human needs. [22].

2. OpeNeed- Ontology for Representing Human Needs [14].

3. GUMO- The General User Model Ontology [30]

4. A Context Model based on Ontological Languages [23]

The user modeling part of this study is created based on the General User Model Ontology (GUMO)[30] [31]. It provides a detailed description of the user, including physiological state, characteristics, personality, demographics, and emotional state.

The context ontology section is derived from the work of Hervás, Bravo, and Fontecha [23]. Concepts from the device model ontology, the environment model ontology, and the information visualization ontology are incorporated in the developed ontology. The Human Needs section is derived from the FHN [22], and OpeNeed Ontologies [14].

\subsection{Conceptualization}

Conceptual Models are essential instruments to represent engineering scenarios. They reflect our understanding of the world and aid in the "development of knowledge about these worlds" [32]. The model presented in Fig.3 is application-dependent; it reflects the idea of a system capable of recognizing human needs and presenting augmented reality experiences that function as satisfiers or means for a satisfier for those needs. This model is based on previous research presented in [17] [18]. 


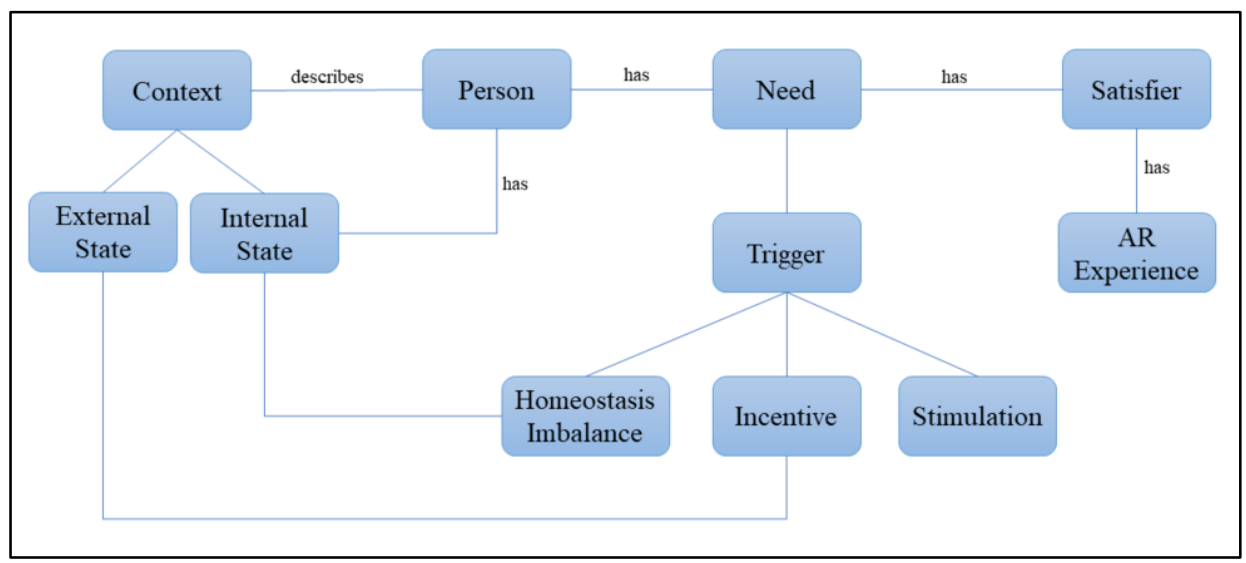

Figure 3. Conceptual Model of Augmented Reality for Human Needs Satisfaction

The main concepts in this model are:

- Person: the user of the system who has a profile and carries sensors to detect his/her basic needs.

- Context: describes the setting/ situation on which a person resides at a certain point in time. Context is divided into external and internal states.

- External State: describes the environment, and all information related to it at a particular time, including temperature, location, activity, social setting.

- Internal State: it portrays the internal state of a person; the change in this state may reflect the occurrence of a need. The main element to be checked in the internal state is the body's homeostasis.

- Need: is one of the basic needs in Max-Neef's model of fundamental human needs. The need is a combination of existential and axiological categories, having a format of Subsistence/Being need, or Subsistence/Having need.

- Trigger: a trigger is a factor that activates a need. There are three types of triggers in psychology [33]. These are Homeostasis imbalance, incentive, and stimulation.

- Homeostasis Imbalance: is the internal state that reflects a malfunction in the body processes resulting in a rise of a need.

- Incentive: is an external positive or negative environmental stimulus that motivates a person.

- Stimulation: is an activity that causes excitement or pleasure.

- Satisfier: an object, idea, or social structure that contributes to the satisfaction of a need.

- Augmented reality experience: is an experience that is developed to function as a satisfier of a basic human need or aid in the satisfaction of the need. 
These concepts describe the problem and the possible solution for it. The ontology development activity of this research is based on this model to discover all related terms and expand the knowledge in this system domain.

\subsection{Enumerated Important Terms in the Ontology}

In addition to the pre-glossary terms identified in the ORSD in section 4.1, further concepts and terms are discovered by creating a Glossary of Terms (GT) that includes concepts, verbs, instances, and properties [24].

Table 2 below shows the most important terms to develop the Ontology within the subjects of Human, Need, Context, Augmented Reality. The list does not reflect the relations between the terms; it just lists them.

Table 2. Important terms in the ontology

\begin{tabular}{crrr}
\hline Human & Need & Context & \multicolumn{1}{c}{$\begin{array}{c}\text { Augmented } \\
\text { Reality }\end{array}$} \\
\hline User & Subsistence & Environment & AR Satisfier \\
Profile & Protection & External State & AR experience \\
Internal State & Affection & Location & Object \\
Hemostasis Imbalance & Understanding & Placement \\
& Participation & Stimulation & System \\
& Leisure & & Direct Satisfier \\
& Creation & & Indirect Satisfier \\
& & & Satisfaction \\
\end{tabular}

\subsection{Define the Classes and Class Hierarchy}

To define the classes and class hierarchy, the Protégé open-source ontology editor [34] is used. A combination of top-down and bottom-up approaches are used for creating classes and connecting them in relations from top-level concepts to details and other times from middle concepts to more general ones (Fig. 4).

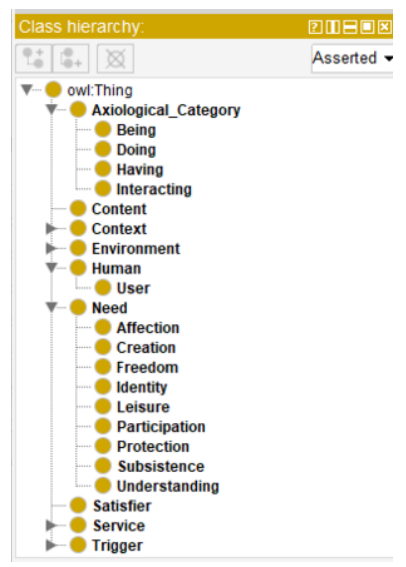

Figure 4 Protégé Class Hierarchy 


\subsection{Define the Properties and facets of Classes-Slots}

The properties of each class describe its structure. In the Protégé editor, there are two types of properties: objects properties that describe the relation between classes and data properties that describe the instances.

Classes can have many facets that describe their features. These include slot cardinality, slot-value type, and domain and range of a slot. For each of the classes identified, defined are the various facets of the properties in the Protégé tool. Each property is then linked to the class it describes (Fig. 5).

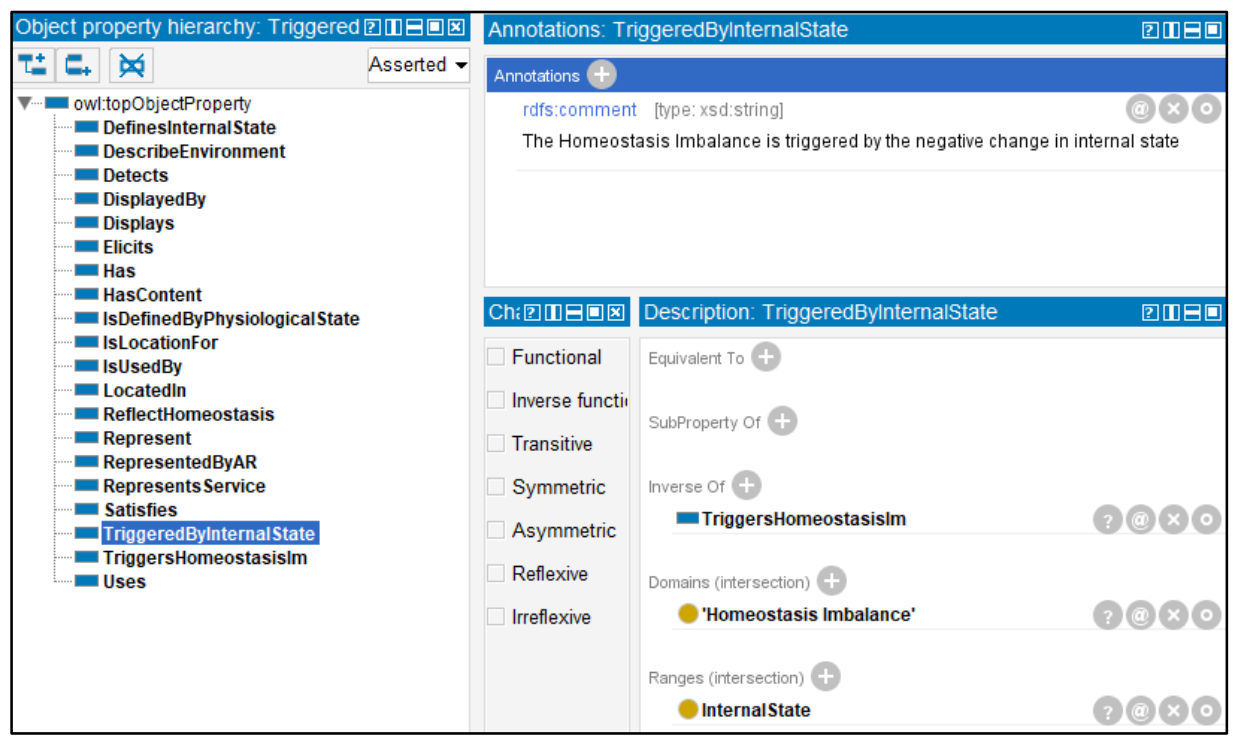

Figure 5 Define Properties and Facets of Classes

\subsection{Create Instances}

The creation of instances helps in answering the many queries the ontology must answer. An essential role of instances is to validate the correct function of the ontology. In Web Protégé, the concept of instances is called individuals. Several instances are created for each of these classes: Human, Need, Satisfier, Augmented Reality experience, and context for ontology evaluation.

\subsection{Ontology Visualization}

To better view the structure and relations in the developed ontology, a visualization is provided (Fig.6) using a web-based visualization tool for ontologies WebVOWL ${ }^{2}$.

\footnotetext{
${ }^{2} \mathrm{http}: / /$ vowl.visualdataweb.org/webvowl.html
} 


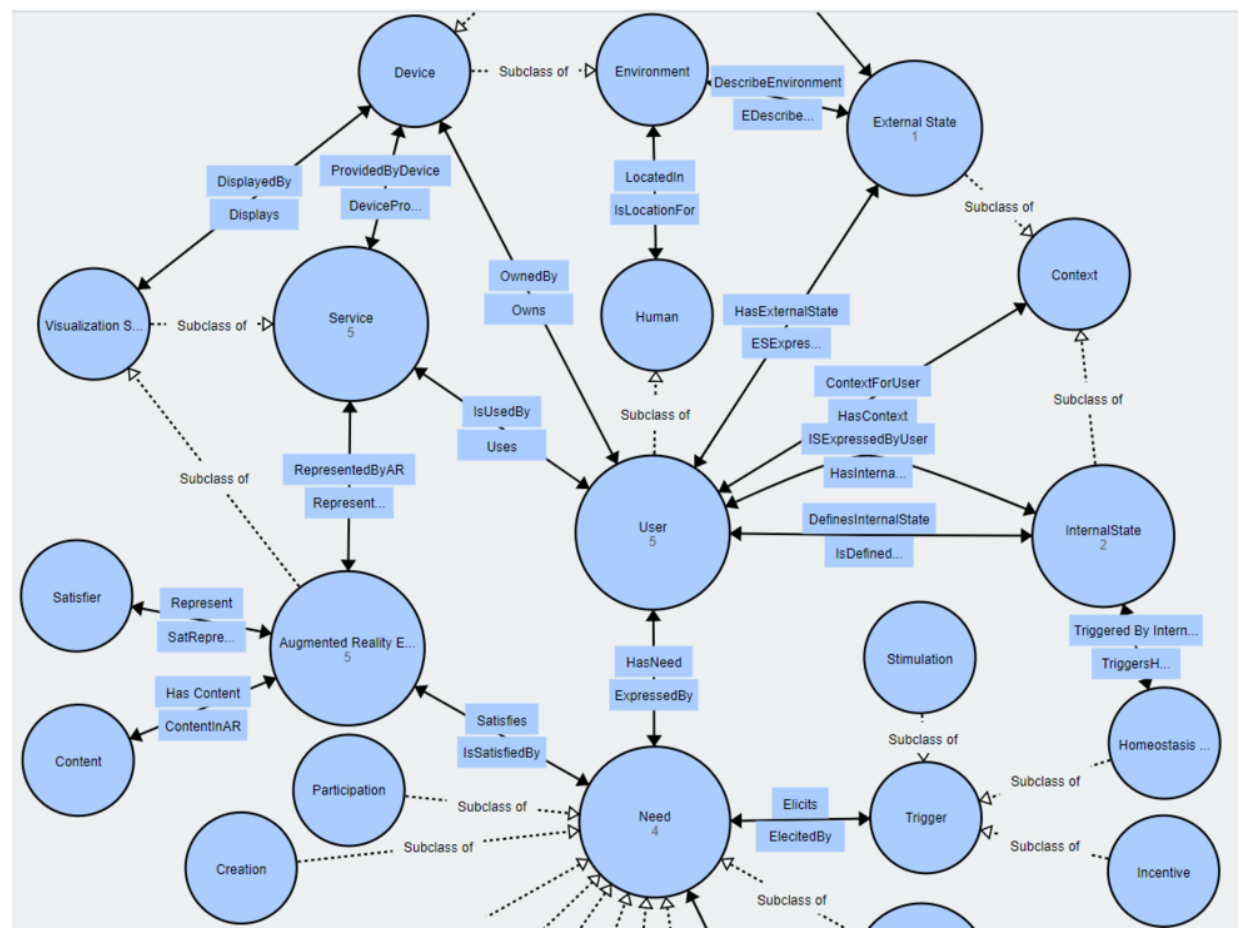

Figure 6 Augmented Reality for Human Needs Ontology Visualization

\section{Evaluation}

Many methods and metrics are available for ontology evaluation. All these methods fall into one of two evaluation categories: verification and validations. While verification is concerned with the correctness of the ontology, validation is concerned with developing the correct ontology [35].

For the evaluation of the AR for Human Needs ontology (AR-HN), the OOPS! Ontology Pitfall Scanner developed by Poveda-Villalon, Suarez-Figueroa, and GomezPerez [36] is used. The OOPS! Ontology Pitfall Scanner is a web-based tool that verifies ontologies' correctness by detecting possible errors. The developers of OOPS! provide a catalog of common pitfalls ${ }^{3}$ that are categorized into the following classification lists [37] :

\section{Evaluation Classification by Dimension:}

- Structural Dimension

- Functional Dimension

- Usability Profiling Dimension

Evaluation Classification by Criteria:

- Consistency

- Conciseness

${ }^{3}$ http://oops.linkeddata.es/catalogue.jsp 


\section{- Completeness}

The list of pitfalls possible to be detected by OOPS! Ontology Pitfall Scanner, according to the abovementioned classification, is presented in Fig.7 and Fig.8, as extracted from [37].

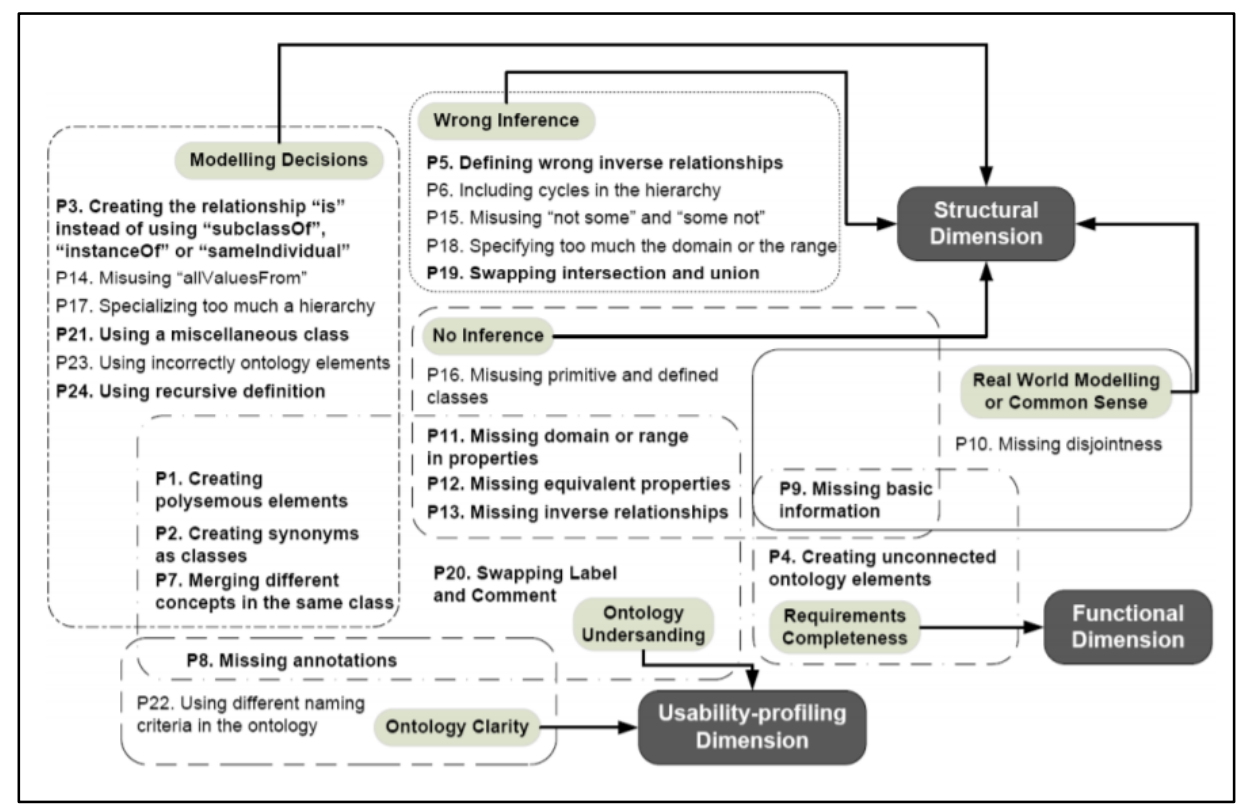

Figure 7 Classification of Pitfalls according to Dimensions [28]

\begin{tabular}{|c|c|c|}
\hline 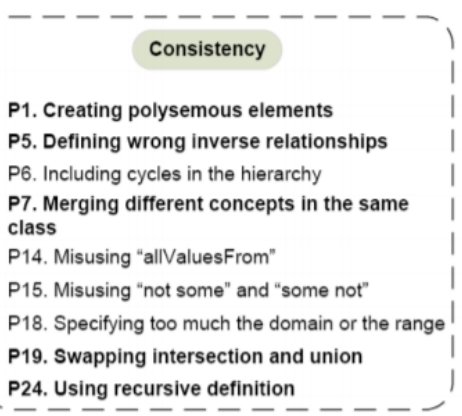 & $\begin{array}{l}\quad \text { Completeness } \\
\text { P4. Creating unconnected } \\
\text { ontology elements } \\
\text { P9. Missing basic information } \\
\text { P10. Missing disjointness } \\
\text { P11. Missing domain or range in } \\
\text { properties } \\
\text { P12. Missing equivalent properties } \\
\text { P13. Missing inverse relationships } \\
\text { P16. Misusing primitive and defined } \\
\text { classes }\end{array}$ & $\begin{array}{l}\quad \text { Conciseness } \\
\text { P2. Creating synonyms } \\
\text { as classes } \\
\text { P3. Creating the relationship "is" } \\
\text { instead of using "subclassOf", } \\
\text { "instanceOf" or "samelndividual" } \\
\text { P17. Specializing too much a } \\
\text { hierarchy } \\
\text { P21. Using a miscellaneous } \\
\text { class }\end{array}$ \\
\hline
\end{tabular}

Figure 8 Classification of Pitfalls according to Criteria [28]

\subsection{Evaluating Correctness of the Ontology: Verification}

Ontology evaluation is an on-going process throughout the design and development stages. In this section, the ontology correctness is verified by comparing the results of 
the scans performed on the Augmented Reality for Human Needs ontology using the OOPS! Ontology Pitfall Scanner. The results of the scan done after the complete ontology development are presented as "AR-HNOnto Previous" in the charts. Alternatively, the results of the scan after addressing the issues detected by the first scan are represented as "AR-HNOnto Final."

The advanced options in OOPS! allows for general and specific scanning by dimension or criteria. For comparisons with the developed ontology, the scanner is being run on two developed ontologies that are named Good Ontologies by the world wide web consortium $\mathrm{W} 3 \mathrm{C}^{4}$ :

- The Marine Ontology

- The Good Relations Ontology

The Marine, Good Relations, and Augmented Reality for Human Needs ontologies are comparable since the size difference is reasonable (Table 3).

Table 3 Sizes of the Compared Ontologies

\begin{tabular}{|l|l|l|}
\hline Ontology & Class Count & Logical Axiom Count \\
\hline Marine & 106 & 267 \\
\hline Good Relations & 38 & 450 \\
\hline Augmented Reality for Human Needs & 32 & 260 \\
\hline
\end{tabular}

\subsubsection{Verification by Dimensions Classification}

This step presents the results of the AR-HN ontology before and after corrections based on the structural, functional, and usability dimensions. The results are compared with the results obtained from the Marine and Good Relations ontologies.

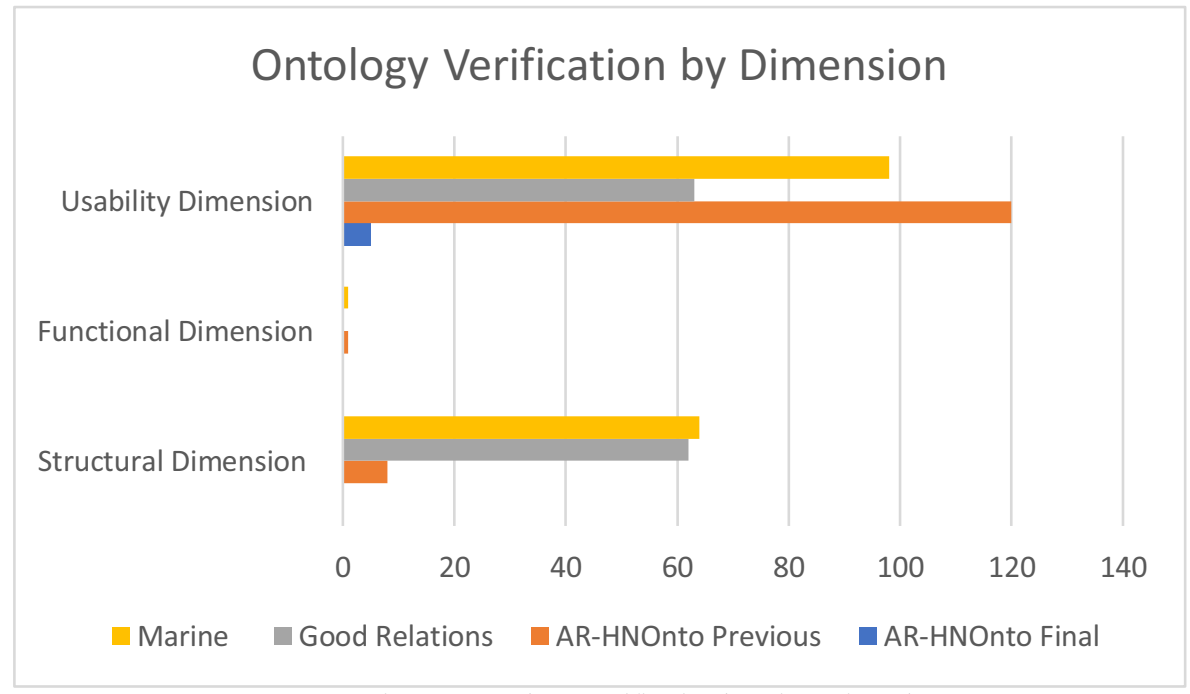

Figure 9 Ontology Verification by Dimension Chart

${ }^{4}$ https://www.w3.org/wiki/Good_Ontologies 
The high number of pitfalls (120 pitfalls) in the usability dimension is due to missing annotations, which aid in the human readability of the ontology. After providing proper annotations in the form of labels and comments, the number of pitfalls decreased significantly.

\subsubsection{Verification by Criteria Classification}

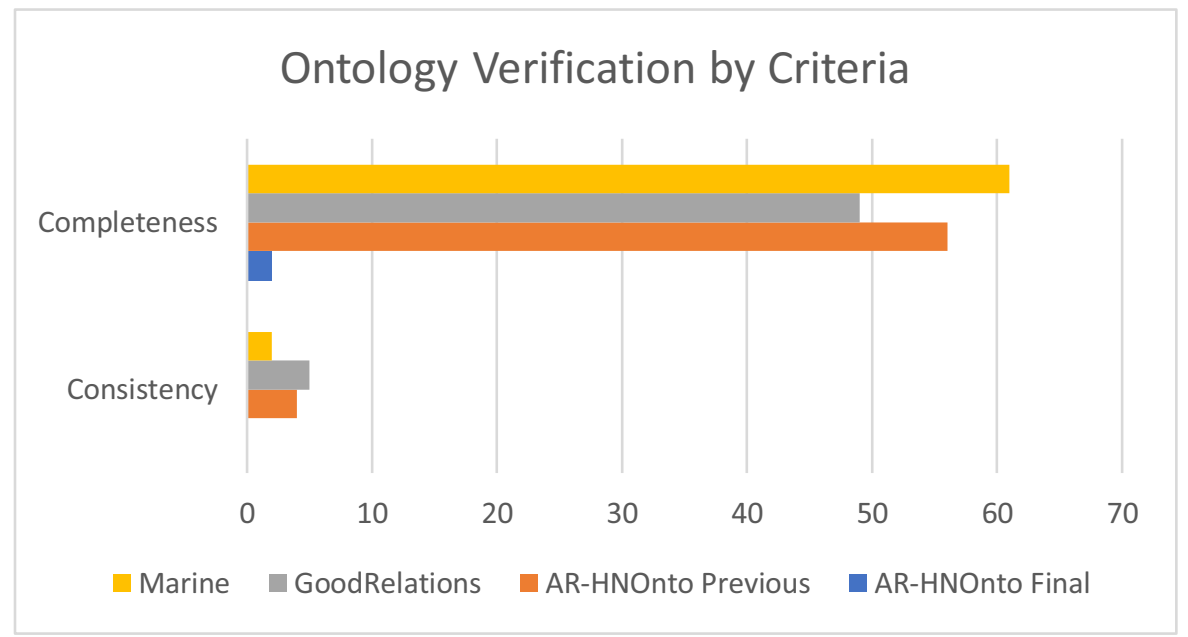

Figure 10 Ontology Verification by Criteria Chart

The results of the Conciseness criteria, which represent the bad practice in the ontology, returned zero pitfalls for all ontologies. Therefore, it is not included in the verification by criteria chart. The consistency result of the AR-HNOnto Previous is four pitfalls, and after correcting it, AR-HNOnto Final has zero consistency pitfalls.

\subsection{Ontology Evaluation via Competency Questions}

The importance of competency questions resides in ensuring the correct performance of the ontology-based on the defined requirements. This section evaluates the ontology based on the competency questions (initially presented in section 4.1):

- Which human need is currently active for a user?

- What augmented reality experience can satisfy a particular need?

- What is the current internal state of the user?

A manual approach is followed to evaluate using the competency questions, as shown in [22]. For that purpose, test data for various class individuals are provided, in addition to a list for expected answers for the competency questions to perform the evaluation.

The first step in this evaluation is to set up the test data; 17 individuals are created as follows:

- $\quad$ Five User Individuals: Adam, Brad, Emma, Kareem, Maya

- Three Context Individuals: InternalContextBalanced, InternalContextImbalanced, ExternalConextImbalanced 
- Four Need Individuals: SubsistenceNeed, AffectionNeed, LeisureNeed, UnderstandingNeed

- $\quad$ Five AR Individuals: ARFoodService, ARHealth Service, AREntertainmentService, ARTransportationService, AREduationalService Then, by setting a total of 14 asserted facts on the individuals. The facts are random, and they reflect a relationship between the primary classes. Table 4 presents the test data, the asserted facts, and the total facts per individual.

Table 4 Test Data Individuals, Asserted, and Inferred Facts

\begin{tabular}{|c|c|c|c|}
\hline Individual & Asserted Facts & $\begin{array}{l}\text { No. of } \\
\text { Asserted } \\
\text { Facts }\end{array}$ & $\begin{array}{l}\text { No. of } \\
\text { Inferred } \\
\text { Facts }\end{array}$ \\
\hline Adam & $\begin{array}{l}\text { HasInternalState }=\text { InternalContext } \\
\text { Imbalanced } \\
\text { HasNeed= SubsistenceNeed } \\
\text { NeedActive = Active }\end{array}$ & 3 & \\
\hline Brad & $\begin{array}{l}\text { HasInternalState }=\text { InternalContext } \\
\text { Balanced }\end{array}$ & 1 & \\
\hline Emma & HasNeed $=$ LeisureNeed & 1 & \\
\hline \multicolumn{4}{|l|}{ Kareem } \\
\hline Maya & $\begin{array}{l}\text { HasNeed }=\text { AffectionNeed } \\
\text { HasNeed }=\text { UnderstandingNeed }\end{array}$ & 2 & \\
\hline InternalContextBalanced & Internal State Balanced & 1 & 1 \\
\hline InternalContextImbalanced & Internal State Imbalanced & 1 & 2 \\
\hline \multicolumn{4}{|l|}{ ExternalContextImbalanced } \\
\hline SubsistenceNeed & NeedActive $=$ Active & 1 & 3 \\
\hline AffectionNeed & & & 1 \\
\hline LeisureNeed & $\begin{array}{l}\text { Is SatisfiedBy }= \\
\text { AREntertainmentService }\end{array}$ & 1 & 1 \\
\hline UnderstandingNeed & & & 2 \\
\hline ARFoodService & Satisfies $=$ SubsistenceNeed & 1 & \\
\hline ARHealthService & Satisfies $=$ SubsistenceNeed & 1 & \\
\hline AREntertainmentService & & & 1 \\
\hline AREducationalService & Satisfies $=$ UnderstandingNeed & 1 & \\
\hline \multicolumn{4}{|l|}{ ARTransportationService } \\
\hline & Total & 14 & 11 \\
\hline
\end{tabular}

The next step is to translate the competency questions into Description Logic (DL) queries to be executed against the ontology. Returning the Expected Answer for each of the competency questions indicates that the ontology meets the requirement.

For DL Queries execution, the Pallet (Incremental) reasoner is used to classify the ontology. Afterward, running the DL queries to retrieve the results.

Competency Question 1: Which human need is currently active for a user?

DL Query: Need and ExpressedBy value Maya 
Expected Answer: a list of needs for a specific user Result:

\begin{tabular}{l} 
DL query: \\
Query (class expression) \\
Need and ExpressedBy value Maya \\
Execute Add to ontology \\
\hline Query results \\
Instances (2 of 2) \\
AffectionNeed \\
UnderstandingNeed \\
\hline
\end{tabular}

Figure 11 Active needs query Execution in Protégé

Competency Question 2: What augmented reality experience can satisfy a specific need? DL Query: AugmentedRealityExperience and Satisfies value SubsistenceNeed Expected Answer: a list of augmented reality services that satisfy the subsistence needs Result:

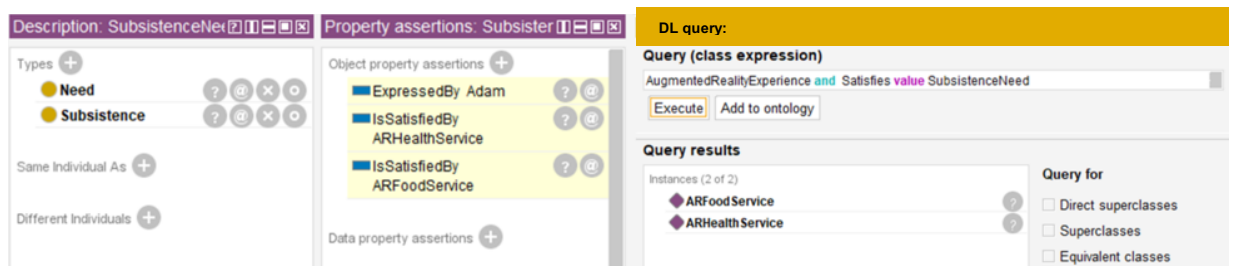

Figure 12 AR experience query execution in Protégé

Competency Question 3: What is the current internal state of the user? DL Query: InternalState and ISExpressedByUser value Emma Expected Answer: the internal state of the user Result:

\begin{tabular}{l} 
DL query: \\
Query (class expression) \\
InternalState and ISExpressedByUser value Emma \\
Execute Add to ontology \\
\hline Query results \\
Instances (1 of 1) \\
InternalContextImbalanced
\end{tabular}

Figure 13 Internal state query execution in Protégé 


\section{Discussion}

Section 5 provides the evaluation of the Augmented Reality for Human Needs ontology for its correctness and completeness using the OOPS! Ontology Pitfall Scanner. Also presented is the evaluation of the ontology via the competency questions to check whether it provides answers to the queries it is meant to answer. The evaluations show positive results. By using the OOPS! Ontology Pitfall Scanner, the tool pinpointed minor, important, and critical pitfalls that are fixed to improve the ontology. The charts in section 5.1, which compare the AR-HN ontology to major ontologies, show that it scored well in the Structural and Functional dimensions. The usability dimension scan detected a high number of pitfalls caused by missing annotations. The completeness criteria contained many pitfalls as well. On the other hand, the consistency criteria returned one pitfall, which is fixed. By addressing the pitfalls, the final ontology is much improved.

Section 5.2 presents the evaluation of competency questions. Seventeen individuals are created, and 14 facts are declared; the Pallet (Incremental) reasoner returned 11 inferred facts. For such a small number of asserted facts, a total of 11 inferred facts is a promising result. The query results of the competency questions reflect correct inference and outcome, which proves that the ontology satisfies the related requirement.

\section{Conclusion and Future work}

This research presents the conceptualization of an OWL vocabulary that defines the Augmented Reality for Human Needs class structure and relationships. The research details the development of the ontology-based on the Methontology [24] and the ontology development guide [21]. The evaluation process for the verification and validation of the ontology is also presented. The augmented reality for human needs ontology shows promising evaluation results. It provides the vocabulary and concepts of relationships for further development of the conceptual model. As a future work and for additional development of the Augmented Reality for Human Needs ontology, the integration with a foundational ontology, to increase interoperability and reusability, is considered.

\section{References}

[1] T.R. Gruber, "'A translation approach to portable ontology specifications," Knowledge acquisition, vol. 5, no. 2, pp. 199-220, 1993.

[2] W3C, "'W3C Semantic Web Vocabulary,", vol. 2020, no. 11 January, 2015.

[3] R. Azuma, Y. Baillot, R. Behringer, S. Feiner, S. Julier and B. MacIntyre, "'Recent advances in augmented reality," IEEE Comput.Graphics Appl., vol. 21, no. 6, pp. 34-47, 2001.

[4] M. Dunleavy and C. Dede, "'Augmented reality teaching and learning,", pp. 735-745, 2014.

[5] J.M. Patricio, M.C. Costa, J.A. Carranca and B. Farropo, "'SolarSystemGO-An augmented reality based game with astronomical concepts,", pp. 1-3, 2018. 
[6] G. Waltner, M. Schwarz, S. Ladstätter, A. Weber, P. Luley, H. Bischof, M. Lindschinger, I. Schmid and L. Paletta, "'Mango-mobile augmented reality with functional eating guidance and food awareness,", pp. 425-432, 2015.

[7] W.S. Khor, B. Baker, K. Amin, A. Chan, K. Patel and J. Wong, "'Augmented and virtual reality in surgery-the digital surgical environment: applications, limitations and legal pitfalls," Annals of translational medicine, vol. 4, no. 23, 2016.

[8] M.A. Frigo, E.C. da Silva and G.F. Barbosa, "'Augmented Reality in Aerospace Manufacturing: A Review."," Journal of Industrial and Intelligent Information, vol. 4, no. 2, 2016.

[9] R. Hammady, M. Ma and N. Temple, "'Augmented reality and gamification in heritage museums,", pp. 181-187, 2016.

[10] G.A. Boy and D. Platt, "'A situation awareness assistant for human deep space exploration,", pp. 629-636, 2013.

[11] D. Han, M.C. tom Dieck and T. Jung, "'User experience model for augmented reality applications in urban heritage tourism," Journal of Heritage Tourism, vol. 13, no. 1, pp. 46-61, 2018.

[12] A. Kaiser and F. Kragulj, "'Bewextra: Creating and inferring explicit knowledge of needs in organizations," Journal of Futures Studies, vol. 20, no. 4, pp. 79-98, 2016.

[13] M.A. Max-Neef, "'Human scale development,", 1991.

[14] S. Human, F. Fahrenbach, F. Kragulj and V. Savenkov, "'Ontology for Representing Human Needs,", pp. 195-210, 2017.

[15] A.K. Dey, "'Understanding and using context," Personal and ubiquitous computing, vol. 5, no. 1, pp. 4-7, 2001.

[16] R. Hervás, J. Bravo, J. Fontecha and V. Villarreal, "'Achieving adaptive augmented reality through ontological context-awareness applied to aal scenarios," Journal of Universal Computer Science, vol. 19, no. 9, pp. 13341349, 2013.

[17] M.A. Yahya, "'A Context-Aware Personalization Model for Augmented Reality Applications ," February, 2016.

[18] M. Yahya and A. Dahanayake, "'A Needs-Based Personalization Model for Context Aware Applications," Frontiers in Artificial Intelligence and Applications, vol. 292, pp. 63-82, 2016.

[19] N. Guarino, G. Guizzardi and J. Mylopoulos, "'On the Philosophical Foundations of Conceptual Models,", 2019.

[20] G. Guizzardi, G. Wagner, J.P.A. Almeida and R.S. Guizzardi, "'Towards ontological foundations for conceptual modeling: The unified foundational ontology (UFO) story," Applied ontology, vol. 10, no. 3-4, pp. 259-271, 2015.

[21] N.F. Noy and D.L. McGuinness, "'Ontology development 101: A guide to creating your first ontology,", 2001.

[22] S.D. Dsouza, "'Cloud-based Ontology Solution for Conceptualizing Human Needs,", 2015.

[23] R. Hervás, J. Bravo and J. Fontecha, "'A Context Model based on Ontological Languages: a Proposal for Information Visualization." J.UCS, vol. 16, no. 12, pp. 1539-1555, 2010.

[24] M. Fernández-López, A. Gómez-Pérez and N. Juristo, "'Methontology: from ontological art towards ontological engineering,", 1997. 
[25] Ni, Pau de la Cruz, Iván and A.B. García Hernando, "'A foundational ontologybased model for human activity representation in smart homes," Journal of Ambient Intelligence and Smart Environments, vol. 8, no. 1, pp. 47-61, 2016.

[26] NeOn Project, "'NeOn Toolkit,", vol. 2020, no. 11 Jan, 2014.

[27] H. Kim, T. Matuszka, J. Kim, J. Kim and W. Woo, "'An ontology-based augmented reality application exploring contextual data of cultural heritage sites,", pp. 468-475, 2016.

[28] I. Horrocks, P.F. Patel-Schneider, H. Boley, S. Tabet, B. Grosof and M. Dean, "SWRL: A semantic web rule language combining OWL and RuleML," W3C Member submission, vol. 21, no. 79, pp. 1-31, 2004.

[29] M. Suárez-Figueroa, A. Gomez-Perez and B. Villazon Terrazas, "'How to Write and Use the Ontology Requirements Specification Document,", vol. 5871, 2009.

[30] D. Heckmann, E. Schwarzkopf, J. Mori, D. Dengler and A. Kröner, "'The user model and context ontology gumo revisited for future web 2.0 extensions," Contexts and Ontologies: Representation and Reasoning, pp. 37-46, 2007.

[31] D. Heckmann, "'Ubiquitous User Modeling,", ed. 297, 2006.

[32] B. Thalheim, "'Conceptual Models and Their Foundations,", pp. 123-139, 2019.

[33] D.G. Myers, "'Psychology,", ed. Tenth edition, international edition, 2013.

[34] Anonymous "'Protege,", vol. 2020, no. 11 Jan.

[35] A. Gomez-Perez, M. Fernández-López and O. Corcho, "'Ontological Engineering: with examples from the areas of Knowledge Management, eCommerce and the Semantic Web,", 2006.

[36] M. Poveda-Villalón, M.C. Suárez-Figueroa and A. Gómez-Pérez, "'Validating ontologies with oops!", pp. 267-281, 2012.

[37] M. Poveda Villalon, M.C. Suárez-Figueroa and A. Gómez-Pérez, "'A double classification of common pitfalls in ontologies,", 2010. 